\title{
Biomassa e coeficiente de utilização biológico de nutrientes pelo Eucalyptus grandis $\times$ E. camaldulensis em diferentes espaçamentos de plantio
}

\author{
Biomass and nutrient biological utilization coefficient of Eucalyptus \\ grandis $\times$ E. camaldulensis in different planting densities
}

\author{
Bruno Oliveira Lafetá ${ }^{10}$, Reynaldo Campos Santana² (D), Gilciano Saraiva Nogueira² (D), \\ Tamires Mousslech Andrade Penido ${ }^{2}$ (1) , Luiz Felipe Ramalho de Oliveira ${ }^{2}$ (1), \\ Diego dos Santos Vieira² (1) \\ ${ }^{1}$ Instituto Federal de Educação, Ciência e Tecnologia de Minas Gerais - IFMG, São João Evangelista, MG, Brasil \\ ${ }^{2}$ Universidade Federal dos Vales do Jequitinhonha e Mucuri - UFVJM, Diamantina, MG, Brasil
}

Como citar: Lafetá, B. O., Santana, R. C., Nogueira, G. S., Penido, T. M. A., Oliveira, L. F. R., \& Vieira, D. S. (2021). Biomassa e coeficiente de utilização biológico de nutrientes pelo Eucalyptus grandis $\times$ E. camaldulensis em diferentes espaçamentos de plantio. Scientia Forestalis, 49(129), e3378. https://doi.org/10.18671/scifor.v49n129.13

\begin{abstract}
Resumo
Informações sobre a produtividade e aspectos nutricionais tem se tornado cada vez mais necessárias para o sucesso da concepção e gestão de povoamentos superadensados. O presente trabalho objetivou avaliar o efeito do espaçamento inicial de plantio na produção de biomassa e no Coeficiente de Utilização Biológico (CUB) de nutrientes por um clone do híbrido Eucalyptus grandis $\times$ E. camaldulensis. O experimento foi estabelecido em blocos ao acaso com três repetições. Os tratamentos foram assim constituídos: T1 - 3,0 × 0,5 m; T2 - 3,0 × 1,0 m; T3 - 3,0 × 1,5 m; T4 - 3,0 × 2,0 m e T5 - 3,0 × 3,0 m. O inventário florestal foi conduzido aos 101 meses de idade, sendo quantificada a biomassa e calculado o CUB dos nutrientes. As estimativas de biomassa no lenho foram de 165, 159, 153, 147 e $134 \mathrm{t} \mathrm{ha}^{-1}$ para T1 a T5, respectivamente. A amplitude de variação do CUB no tronco foi de 923 a 1174, de 7573 a 10450, de 948 a 1089, de 657 a 894, de 4807 a 6118 e de 2611 a 3497 kg de matéria seca por kg de N, P, K, Ca, Mg e S, respectivamente. Conclui-se que a influência do espaçamento no CUB da casca, lenho e tronco de povoamentos de eucalipto se diferencia entre nutrientes. $O$ espaçamento mais adensado exibiu menores estimativas de CUB para $\mathrm{P}$ e $\mathrm{Mg}$ no lenho. $\mathrm{O}$ espaçamento mais amplo apresentou o menor CUB de $\mathrm{S}$ no lenho e o maior CUB de $\mathrm{K}$ na casca. Espaçamentos adensados apresentam fustes menores e, quando devidamente colhidos na idade técnica de corte, são mais produtivos em unidade de área.
\end{abstract}

Palavras-chave: CUB; Densidade de plantio; Florestas energéticas.

\begin{abstract}
Information about productivity and nutritional aspects has become increasingly necessary for successful conception and management of dense stands. The aim of this research was to evaluate the effect of initial plant spacing on biomass production and Biological Utilization Coefficient (BUC) of nutrients by a hybrid clone of Eucalyptus grandis $\times$ E. camaldulensis. The experiment was established in a randomized three blocks design. The treatments were: T1 - $3.0 \times 0.5 \mathrm{~m} ; \mathrm{T} 2-3.0 \times 1.0 \mathrm{~m} ; \mathrm{T} 3-3.0 \times 1.5 \mathrm{~m} ; \mathrm{T} 4-3.0 \times 2.0 \mathrm{~m}$ and T5 $-3.0 \times 3.0 \mathrm{~m}$. A forest inventory was conducted at 101 months of age, with quantified biomass and BUC of nutrient calculated. Wood biomass estimates were 165, 159, 153, 147 and $134 \mathrm{t} \mathrm{ha}^{-1}$ for T1 to T5, respectively. The variation range of trunk BUC was from 923 to 1174, from 7573 to 10450, from 948 to 1089, from 657 to 894 , from 4807 to 6118 and from 2611 to $3497 \mathrm{~kg}$ of dry matter per kg of N, P, K, Ca,
\end{abstract}

Fonte de financiamento: IFMG.

Conflito de interesse: Nada a declarar.

Autor correspondente: bruno.lafeta@ifmg.edu.br

Recebido: 16 maio 2019.

Aceito: 26 março 2020

Editor: Paulo Henrique Müller Silva.

(c) (†) Este é um artigo publicado em acesso aberto (Open Access) sob a licença Creative Commons Attribution, que permite uso, distribuição e reprodução em qualquer meio, sem restrições desde que o trabalho original seja corretamente citado. 
$\mathrm{Mg}$, and S, respectively. It was concluded that the spacing influence on BUC of bark, wood and trunk of eucalyptus stands is different for nutrients. The denser spacing exhibited lower estimates of BUC for P and $\mathrm{Mg}$ in wood. The widest spacing presented the smallest BUC of $\mathrm{S}$ in wood and largest BUC of $\mathrm{K}$ in bark. Dense spacings have smaller stems and, when properly harvested at the technical cutting age, are more productive per unit of area.

Keywords BUC; Planting density; Energy forests.

\section{INTRODUÇÃO}

A qualidade e a produtividade de povoamentos florestais são fatores determinantes para a conquista de bons mercados e obtenção de fluxo fabril contínuo de matéria-prima. As maiores produtividades no Brasil são atribuídas aos avanços nas pesquisas e desenvolvimento de novas tecnologias. Na década de 70, as plantações de Eucalyptus apresentavam incremento em torno de $15 \mathrm{~m}^{3} \mathrm{ha}^{-1}$ ano-1 $^{-1}$, após 35 anos, esse valor triplicou por meio de avanços silviculturais no preparo do solo, melhoramento genético, metodologia de propagação, fertilização e controle de pragas (Stape et al., 2010).

O cultivo de espécies florestais está sujeito a uma série de fatores ecológicos que afetam de forma direta o crescimento e produção madeireira, podendo ser classificados como abióticos e bióticos (Zhou et al., 2018; Tavares et al., 2019). As propriedades que determinam a qualidade ou produtividade de um local são naturais e inerentes ao mesmo, mas podem ser influenciados pelas práticas de manejo adotadas como a densidade do plantio (Resquin et al., 2019).

A demanda de recursos que influenciam a produtividade (água, luz, nutrientes, energia radiante, temperatura e gás carbônico) pode ser distinta entre árvores, acentuando-se ao longo do tempo em virtude da competição (Binkley et al., 2010). O maior limitante do crescimento de monocultivos no país é a disponibilidade hídrica anual (Stape et al., 2010; Martins et al., 2018). Tal limitante é substancialmente potencializado com o aumento da densidade de plantio. A forte competição por luz e água em florestas energéticas influencia a eficiência de uso desses recursos, promovendo a ocorrência de fustes de menor porte, mesmo que haja disponibilidade de nutrientes provenientes de fertilizações (Schwerz et al., 2019).

No caso de florestas energéticas, que utilizam espaçamentos reduzidos, conciliar a limitação de espaço a ser explorado por uma árvore, o incremento na produtividade, qualidade da madeira, rotações mais curtas e uso sustentável dos recursos é um grande desafio para a silvicultura. A densidade de plantio exerce influência sobre o crescimento de plantas e na qualidade da madeira (Rocha et al., 2016). Plantios em espaçamentos mais adensados são importantes em termos energéticos, pois tendem a estagnar a taxa de crescimento e atingir o potencial produtivo em idades mais juvenis. Em contrapartida, devido às rotações mais curtas, o manejo silvicultural torna-se mais intensivo e reduz os benefícios provenientes do cultivo mínimo, prática comum em povoamentos florestais (Novais et al., 2007; Ferreira et al., 2016; Leslie et al., 2020).

O emprego de sucessivas rotações e altas produtividades impõem elevada exportação e demanda de nutrientes sobre o sítio; a reposição de nutrientes exportados é crucial para manter o sistema sustentável (Eufrade Junior et al., 2016). Estudos que avaliam a absorção de nutrientes pelas plantas e sua relação com a produção de biomassa, ou seja, sua eficiência de uso, são úteis para a definição de procedimentos que minimizam a exportação de nutrientes após colheita florestal.

A eficiência de utilização de nutrientes, definida como coeficiente de utilização biológico dos nutrientes (CUB), pode ser usada para auxiliar a seleção de materiais genéticos indicados para diferentes condições edáficas e climáticas e para a recomendação de fertilizantes (Barros et al., 1986, 1995). É calculada pela razão da quantidade de matéria seca produzida por grama de nutriente ou, simplesmente, o inverso da concentração do nutriente nos tecidos. 
O CUB e a expectativa de produtividade são informações que permitem a estimativa da quantidade de nutrientes necessária para um adequado balanço nutricional da próxima rotação. A recomendação de fertilização pode ser realizada fundamentando-se em diferentes métodos. O balanço nutricional é o método mais utilizado para a estimativa da quantidade de nutrientes em cultivos de eucalipto (Lafetá et al., 2018). Os processos que influenciam o CUB estão relacionados com características morfológicas (sistema radicular eficiente, alta relação raiz-parte aérea e sistema radicular extensivo) e fisiológicas (taxa fotossintética e a capacidade de manter o metabolismo normal com baixo teor de nutrientes nos tecidos) (Novais et al., 2007).

Embora a regulação seja tradicionalmente planejada por empresas com extensas áreas de cultivo, não é difícil encontrar talhões além da idade técnica de corte devido a restrições de colheita, como aspectos econômicos, distância (Binoti et al., 2014) e/ou inacessibilidade em determinadas épocas do ano. A quantificação de nutrientes exportados via colheita, mesmo em de idades além daquelas convencionalmente adotadas para o corte de eucalipto no país, entre 6 e 8 anos, subsidiam a racionalização do uso de corretivos e fertilizantes em rotações subsequentes, importante para a silvicultura de precisão e sustentabilidade do ecossistema. O emprego de práticas silviculturais apropriadas associado ao uso de métodos de avaliação nutricional é uma alternativa viável na busca de informações para melhor planejamento da fertilização e aumento da produtividade. Portanto, o presente trabalho objetivou avaliar o efeito do espaçamento inicial de plantio na produção de biomassa e no coeficiente de utilização biológico de nutrientes pelo Eucalyptus grandis $\times$ E. camaldulensis, aos 101 meses de idade.

\section{MATERIAL E MÉTODOS}

O estudo foi conduzido a $17^{\circ} 50^{\prime}$ de latitude sul e $42^{\circ} 49^{\prime}$ de longitude oeste, no município de Itamarandiba-MG. O clima predominante na região é do tipo Cwa, segundo a classificação de Köppen (Köppen, 1936), com verões quentes e chuvosos e invernos amenos e secos com estação seca bem definida de abril a setembro. A precipitação média anual é de $1034 \mathrm{~mm}$ e a temperatura média anual de $19,7^{\circ} \mathrm{C}$; em termos médios, novembro é o mês mais chuvoso (223 mm) e julho, o mais seco $(6 \mathrm{~mm})$. Os dados climáticos foram provenientes de registros anuais de 1961 a 1990, disponíveis no banco de dados do Instituto Nacional de Meteorologia (2019).

O experimento foi instalado em dezembro de 2002 utilizando-se um clone híbrido de Eucalyptus grandis W. Hill ex Maiden $\times$ Eucalyptus camaldulensis Dehnh em, pela classificação da Empresa Brasileira de Pesquisa Agropecuária (2018), Latossolo VermelhoAmarelo com relevo plano e a $1097 \mathrm{~m}$ de altitude. Na fase de implantação, foram realizadas operações de roçada, capina química, subsolagem/fosfatagem, combate a formiga, coveamento manual, plantio, fertilização de plantio com NPK e adubação de cobertura com $\mathrm{KCl}$; detalhes sobre a fertilização em cada etapa de implantação estão descritos em Müller et al. (2005a, 2005b).

Adotou-se delineamento em blocos ao acaso com três blocos, sendo estudado o efeito de cinco tratamentos constituídos pelos seguintes espaçamentos de plantio: T1) 3,0 × 0,5 m6667 plantas ha-1; T2) 3,0 × 1,0 m - 3333 plantas ha-1; T3) 3,0 × 1,5 m- 2222 plantas ha-1; T4) 3,0 $\times 2,0 \mathrm{~m}-1667$ plantas ha-1 e T5) 3,0 × 3,0 m- 1111 plantas ha-1; sendo $3 \mathrm{~m}$ a distância fixa entre linhas de plantio. Cada parcela experimental foi constituída de seis linhas de plantio e em cada linha foram plantadas 28 mudas, perfazendo um total de 168 indivíduos por tratamento. Mensurou-se 48 árvores em cada parcela por ter sido adotada a bordadura dupla entre parcelas. Os percentuais de mortalidade aos 48 meses de idade foram de $3 \%, 4 \%, 1 \%$, $3 \%$ e $2 \%$ em T1 a T5, respectivamente.

O inventário florestal foi realizado aos 101 meses de idade. A escolha dessa idade se pautou em aspectos de regulação e colheita da rotina operacional de uma empresa florestal. Mensuraram-se o diâmetro com casca à 1,30 m de altura (DAP, cm) e altura total $(\mathrm{HT}, \mathrm{m})$ de todos os indivíduos com auxílio de suta e clinômetro Suunto, respectivamente. Foram 
abatidas 50 árvores por espaçamento conforme classe diamétrica, amplitude de $5 \mathrm{~cm}$, para realização da cubagem rigorosa pelo método de Smalian com as seguintes posições de medição: 0,10 m; 0,30 m; 0,70 m; 1,30 m; 2,00 m e assim sucessivamente a cada 2,00 m, até a altura comercial (diâmetro com casca de $4 \mathrm{~cm}$ ). A espessura da casca foi medida com paquímetro digital de precisão $0,01 \mathrm{~mm}$. Os percentuais de mortalidade identificados nesse inventário foram de 13\%, 13\%, 9\%, 11\% e 11\% em T1 a T5, respectivamente.

Estimou-se a biomassa de lenho até a altura comercial ( $\mathrm{ha}^{-1}$ ), sem galhos, para todas as árvores mensuradas nos inventários, empregando a relação multiplicativa entre Volume Sem Casca (VSC) e a Densidade Básica da madeira (DB). O VSC foi estimado pelo ajuste do modelo linearizado de Schumacher e Hall (1933) (Tabela 1). Retiraram-se discos de $6 \mathrm{~cm}$ de espessura a $0 \%$ (base), 25\%, 50\%, 75\% e 100\% (topo) da altura comercial de uma árvoreamostra (aquela com diâmetro médio quadrático) por unidade experimental, totalizando 15 árvores. De cada disco foram obtidas cunhas opostas, que foram utilizadas para a determinação da DB de acordo o método de imersão em água, segundo a norma ABNT NBR 11941 (Associação Brasileira de Normas Técnicas, 2003). As densidades básicas foram de $0,524 \pm 0,006 \mathrm{~g} \mathrm{~cm}^{-3}, 0,554 \pm 0,001 \mathrm{~g} \mathrm{~cm}^{-3}, 0,559 \pm 0,003 \mathrm{~g} \mathrm{~cm}^{-3}, 0,564 \pm 0,003 \mathrm{~g} \mathrm{~cm}^{-3}$ e 0,546 $\pm 0,023 \mathrm{~g} \mathrm{~cm}^{-3}$ para T1 a T5, respectivamente. Nesta mesma sequência, o DAP das árvoresamostra foram de 8,467 $\pm 0,058 \mathrm{~cm}, 10,767 \pm 0,569 \mathrm{~cm}, 12,467 \pm 0,231 \mathrm{~cm} .13,767 \pm 0,651$ $\mathrm{cm}$ e $15,533 \pm 0,231$.

Tabela 1. Equações utilizadas para estimativa do volume sem casca e valores médios encontrados para a densidade básica de eucalipto para diferentes espaçamentos de plantio (Esp.) aos 101 meses de idade.

\begin{tabular}{|c|c|c|c|}
\hline Esp & Equações & $\overline{\boldsymbol{R}}^{2}$ & Syx \\
\hline $3,0 \times 0,5 \mathrm{~m}$ & $\mathrm{LnVSC}=-9,966^{*}+2,278^{*} \mathrm{LnDAP}+0,702^{*} \mathrm{LnHt}$ & 0,995 & 0,05 \\
\hline $3,0 \times 1,0 \mathrm{~m}$ & $\operatorname{LnVSC}=-10,103^{*}+1,958^{*} \mathrm{LnDAP}+0,987^{*} \mathrm{LnHt}$ & 0,991 & 0,08 \\
\hline $3,0 \times 1,5 \mathrm{~m}$ & LnVSC $=-10,326^{*}+1,944^{*} \mathrm{LnDAP}+1,069^{*} \mathrm{LnHt}$ & 0,994 & 0,05 \\
\hline $3,0 \times 2,0 \mathrm{~m}$ & $\operatorname{LnVSC}=-10,569^{*}+1,745^{*} \operatorname{LnDAP}+1,309^{*} \mathrm{LnHt}$ & 0,989 & 0,05 \\
\hline $3,0 \times 3,0 \mathrm{~m}$ & LnVSC $=-10,787^{*}+1,644^{*} \mathrm{LnDAP}+1,454^{*} \mathrm{LnHt}$ & 0,983 & 0,16 \\
\hline
\end{tabular}

*significativo a 5\% de probabilidade pelo teste t; DAP $(\mathrm{cm}) ; \mathrm{Ht}(\mathrm{m})$. VSC = volume sem casca $\left(\mathrm{m}^{3}\right) . \bar{R}^{2}=$ coeficiente de determinação ajustado. $S_{y x}=$ erro-padrão da estimativa.

A biomassa de casca ( $\left(\mathrm{ha}^{-1}\right)$ foi estimada a partir de sua proporcionalidade com a aquela de lenho. As mesmas árvores-amostra tiveram a casca pesada em campo e subamostras foram coletadas em toda extensão longitudinal do tronco e secas a $65^{\circ} \mathrm{C}$ até massa constante em estufa com circulação forçada de ar. As proporções de biomassa de casca com aquela de lenho foram de $9,27 \%, 8,76 \%, 8,76 \%, 7,61 \%$ e $7,76 \%$ para os tratamentos $\mathrm{T} 1, \mathrm{~T} 2$, T3, T4 e T5, respectivamente.

A análise química de rotina foi realizada após coleta de subamostras de casca e lenho distribuídas em toda extensão longitudinal do tronco das árvores-amostra conforme Empresa Brasileira de Pesquisa Agropecuária (2000). O material vegetal foi moído em moinho de facas tipo Willey, em peneira com malha de $1,0 \mathrm{~mm}$, e submetido à digestão nítrico-perclórica. Os teores de $\mathrm{P}$ foram determinados por colorimetria, de $\mathrm{K}$ por fotometria de emissão de chama, de S por turbidimetria, Ca e Mg por espectrofotometria de absorção atômica. O N total foi determinado pelo método Kjeldhal após digestão sulfúrica.

As árvores-amostra foram utilizadas para a estimativa de biomassa e conteúdo de nutrientes na casca e lenho para as demais árvores inventariadas. Esta decisão se pautou na premissa de que existe pouca variação dos teores de nutrientes entre classes de diâmetro; decisão também adotada por Santana et al. (2002) e Habitzreiter et al. (2019). Calculou-se o CUB da casca, lenho e tronco em hectare ( $\mathrm{kg}$ de matéria seca por $\mathrm{kg}$ de nutrientes, que reflete 
o inverso do teor de nutrientes), conforme recomendações de Barros et al. (1986) e Barros et al. (1995).

Para a construção de histogramas de frequências, as informações de DAP e Ht foram agrupadas em classes com intervalos regulares de $2 \mathrm{~cm}$ e $2 \mathrm{~m}$, respectivamente. Calcularamse a assimetria, curtose e correlação linear de Pearson ( $r$ ) entre estas duas métricas com a distância entre plantas. O método dos momentos foi usado no cálculo dessas duas primeiras estatísticas.

Os dados de biomassa e CUB foram submetidos aos testes de normalidade segundo Shapiro-Wilk, homogeneidade de variâncias por Bartlett, autocorrelação por Durbin-Watson e aditividade por Tukey. Para avaliar o efeito do espaçamento inicial de plantio, realizaram-se análises de variância (teste F) e regressão. Testaram-se modelos polinomiais de primeiro e segundo grau pelo método dos Mínimos Quadrados Ordinários (MQO) para a estimativa de biomassa e CUB em função da distância entre plantas. A seleção dos melhores modelos para as análises gráficas subsequentes se embasou na significância dos parâmetros pelo teste $t$, teste de falta de ajuste (lack of fit), maior coeficiente de determinação ajustado ( $\bar{R}^{2}$ ) e menores valores do erro-padrão da estimativa $\left(S_{y x}\right)$ e do critério de informação de Akaike (Akaike Information Criterion, AIC).

As análises estatísticas foram efetuadas com auxílio do software $R$ versão 3.5.2 (R Core Team, 2018), por meio dos pacotes estatísticos alr3 (Weisberg, 2018), ExpDes.pt (Ferreira et al., 2018), MASS (Venables \& Ripley, 2002) e stats (R Core Team, 2018), com significância de 1 e 5\% de probabilidade.

\section{RESULTADOS E DISCUSSÃO}

A densidade de plantas influenciou o crescimento em diâmetro e em altura. As árvores foram maiores nos plantios mais espaçados; as distribuições biométricas se encontraram deslocadas mais à direita nos maiores espaçamentos (Figura 1). Os dois maiores espaçamentos $(3,0 \times 2,0 \mathrm{~m} \mathrm{e} \mathrm{3,0} \mathrm{\times 3,0} \mathrm{m)} \mathrm{apresentaram} \mathrm{concentração} \mathrm{de} \mathrm{árvores}$ em duas classes diamétricas que destoaram daquelas adjacentes. Este fato é de grande importância prática quando se prevê a colheita mecanizada, maiores rendimentos operacionais são esperados em áreas homogêneas e de maior diâmetro (Spinelli et al., 2009).

A competição aumentou a frequência de árvores menores nos espaçamentos mais adensados, corroborando com os resultados observados por Tenorio et al. (2018) e Schwerz et al. (2019). É provável que a intensa competição no espaçamento 3,0 × 0,5 m tenha provocado mudanças na forma das distribuições de DAP. O valor de assimetria das distribuições de DAP foram de 0,08 (curtose $=2,53$ ), $-0,76$ (curtose $=4,51$ ), $-0,69$ (curtose $=$ 3,67 ), $-0,90$ (curtose $=4,81$ ) e $-2,03$ (curtose $=8,79$ ) para os tratamentos T1, T2, T3, T4 e T5, respectivamente. Nesta sequência, para a altura, os valores foram de $-0,80$ (curtose $=$ $3,96),-2,60$ (curtose $=14,76),-1,86$ (curtose $=8,50),-1,87$ (curtose $=8,34$ ) e $-4,20$ (curtose $=24,15)$.

Árvores maiores em povoamentos equiâneos crescem mais rápido que aquelas menores, pois interceptam mais luz e são mais eficientes no uso dos recursos disponíveis no meio (Binkley et al., 2013; Campoe et al., 2013a, 2013b). A predominância de árvores maiores, que apresentam taxas de crescimento superiores às menores (suprimidas), repercutiu em assimetrias negativas (com cauda à esquerda) nos espaçamentos mais amplos. Ressalta-se que assimetrias negativas são comuns em povoamentos equiâneos (Binoti et al., 2010). As distribuições de DAP apresentaram menores valores de assimetria $(r=-0,95 ; p=0,0137)$ e exibiram maior coeficiente de curtose $(r=0,92 ; p=0,0271)$ à medida que aumentou a distância entre plantas. No que se refere à altura, não foi constatada correlação significativa da assimetria e curtose com a distância entre plantas. 

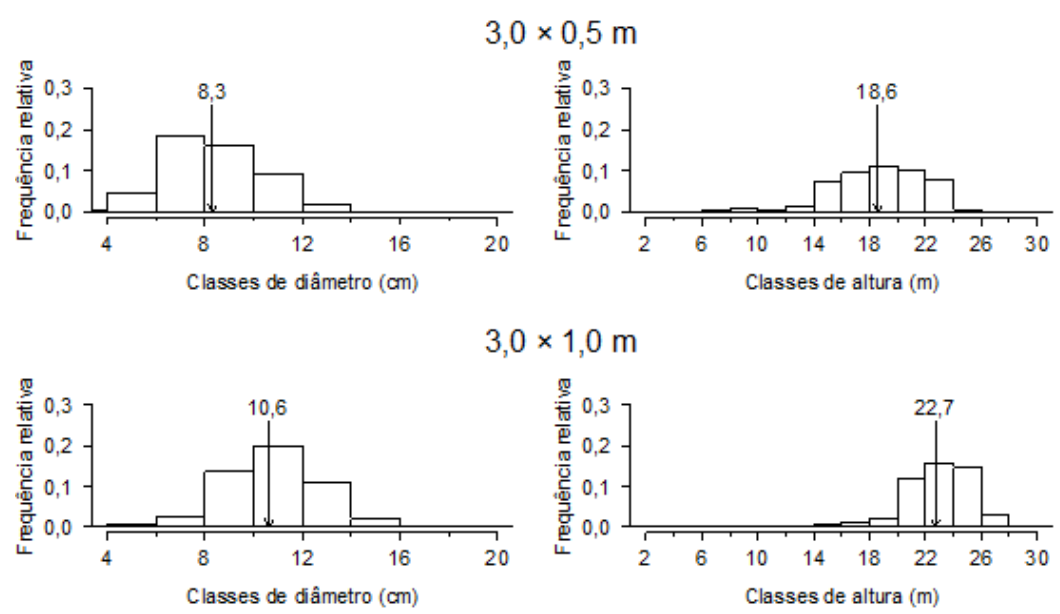

$3,0 \times 1,0 \mathrm{~m}$
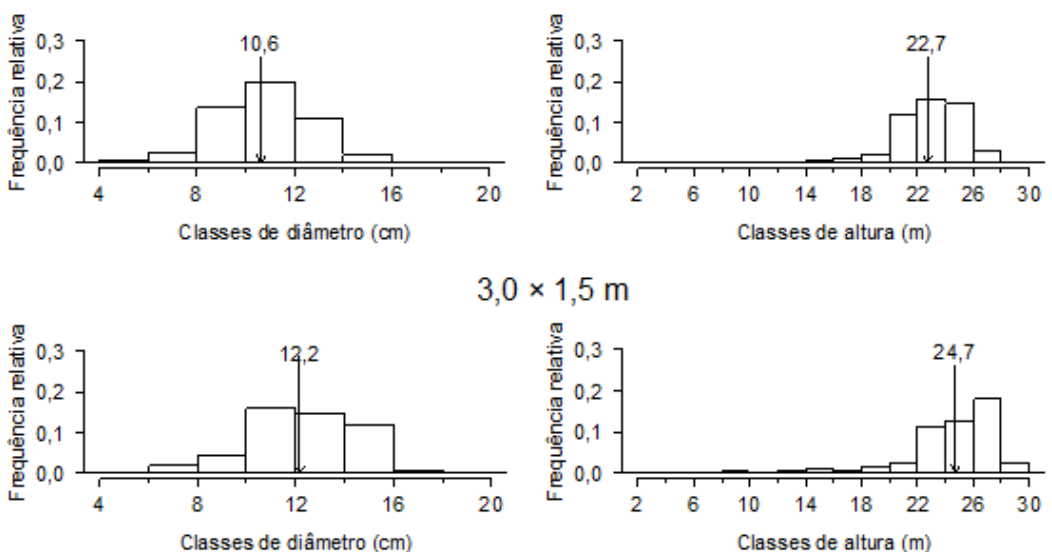

$3,0 \times 1,5 \mathrm{~m}$

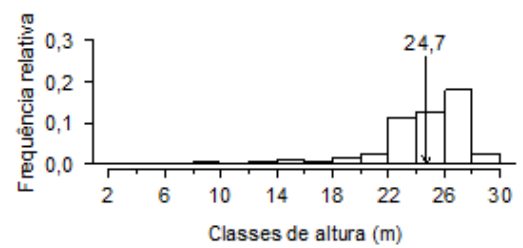

$3,0 \times 2,0 \mathrm{~m}$
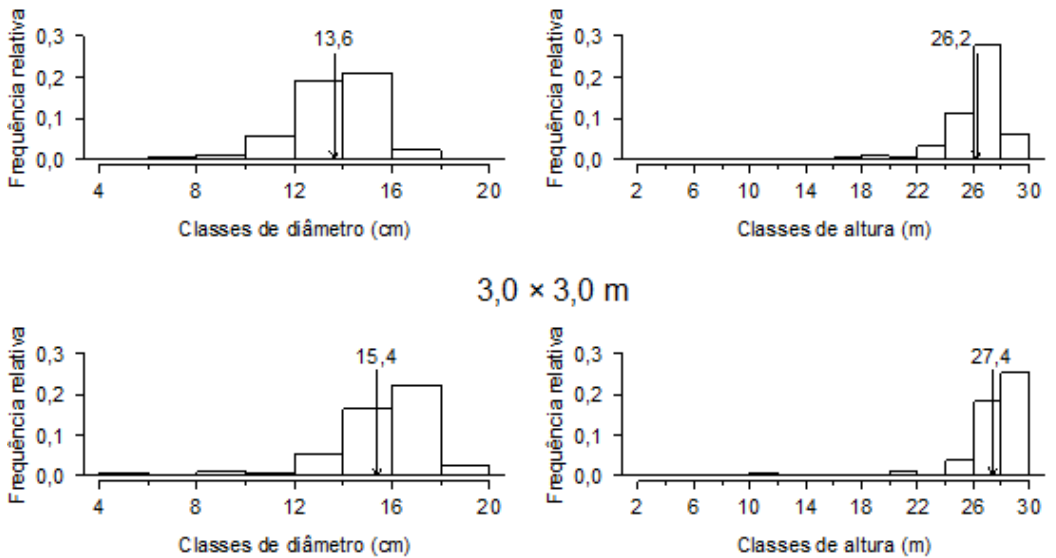

$3,0 \times 3,0 \mathrm{~m}$

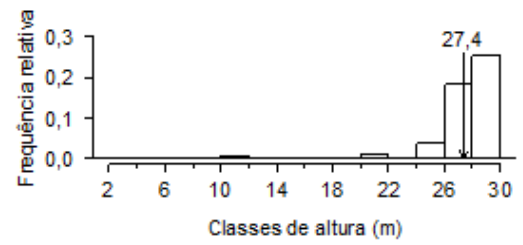

Figura 1. Frequências relativas observadas por classe diamétrica e de altura para diferentes espaçamentos de eucalipto aos 101 meses idade.

No que se refere aos dados de biomassa e CUB, as premissas de normalidade, homogeneidade de variâncias, independência de resíduos e aditividade foram atendidas ( $p$ > 0,05). O resumo da análise de variância se encontra na Tabela 2 . Os espaçamentos de plantio influenciaram todos os atributos avaliados, proporcionando um uso diferenciado de nutrientes absorvidos pelo genótipo. Os coeficientes de variação foram baixos e evidenciaram a precisão experimental.

A Tabela 3 apresenta as equações selecionadas para as estimativas de biomassa e CUB para diferentes espaçamentos de eucalipto, em função da distância entre plantas. Todos os parâmetros foram significativos pelo teste $t(p \leq 0,05)$, comprovando a dependência da biomassa e do CUB para variações do espaçamento. As equações contidas nessa tabela não apresentaram falta de ajuste $(p>0,01)$, exceto aquelas relacionadas ao CUB de Ca no lenho e tronco, e de $S$, somente no lenho. Em geral, os ajustes dos modelos polinomiais testados denotaram adequabilidade para elucidar o comportamento das variáveis dependentes em diferentes espaçamentos de plantio. As equações para a estimativa de atributos pertinentes à casca tiveram os menores coeficientes de determinação ajustado, porém todas as estimativas de suas relações funcionais se aderiram às observações. 
Tabela 2. Resumo da análise de variância para biomassa e Coeficiente de Utilização Biológico (CUB) de nutrientes para diferentes espaçamentos de eucalipto aos 101 meses idade.

\begin{tabular}{|c|c|c|c|c|c|c|}
\hline \multirow{2}{*}{ Atributo } & Casca & Lenho & Tronco & Casca & Lenho & Tronco \\
\hline & \multicolumn{3}{|c|}{ Quadrados médios dos tratamentos } & \multicolumn{3}{|c|}{$\mathrm{CV}_{\exp }(\%)$} \\
\hline Biomassa & $1,10^{*}$ & $513,11 *$ & $629,89 *$ & 8,86 & 6,77 & 6,90 \\
\hline $\mathrm{CUB}_{\mathrm{N}}$ & $6556,12^{* *}$ & $116896,80 * *$ & $17497,69 * *$ & 5,35 & 2,84 & 2,07 \\
\hline CUB $_{\mathrm{P}}$ & $4437533,00 * *$ & $1569502,00 * *$ & $3190917,00 * *$ & 13,29 & 1,64 & 2,55 \\
\hline $\mathrm{CUB}_{\mathrm{K}}$ & $7990,29 * *$ & $13485,13^{* *}$ & $6002,06 * \star$ & 6,95 & 1,78 & 1,94 \\
\hline $\mathrm{CUB}_{\mathrm{Ca}}$ & $655,50 * \star$ & $241485,00 * \star$ & $25952,39 * *$ & 5,79 & 2,20 & 2,96 \\
\hline $\mathrm{CUB}_{\mathrm{Mg}}$ & $37028,78^{* *}$ & $772801,40 * *$ & $583771,40 * *$ & 5,62 & 1,66 & 2,01 \\
\hline $\mathrm{CUB}_{\mathrm{s}}$ & $89283,44^{*}$ & $285009,20 * *$ & $243114,70 * *$ & 4,85 & 2,30 & 2,36 \\
\hline
\end{tabular}

*,** significativo a 5 e $1 \%$ de probabilidade pelo teste $\mathrm{F}$, respectivamente. $\mathrm{CV}_{\exp }=$ Coeficiente de variação experimental. Os graus de liberdade foram de 2,4 e 8 , para bloco, tratamento e resíduo, respectivamente.

Tabela 3. Equações selecionadas para a estimativa da biomassa (t ha-1) e coeficiente de utilização biológico (CUB) para diferentes espaçamentos de eucalipto aos 101 meses idade, em função da distância entre plantas $(D, m)$.

\begin{tabular}{|c|c|c|c|c|}
\hline Var. & Equações & $\overline{\boldsymbol{R}}^{2}$ & $S_{y x}$ & AIC \\
\hline \multicolumn{5}{|c|}{ 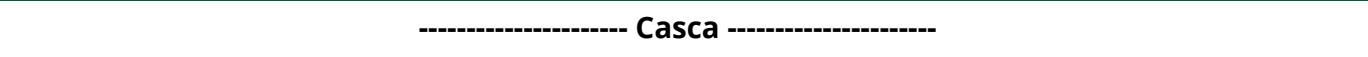 } \\
\hline Bio & Biomassa $=14,9664^{* *}-1,3346^{*} D$ & 0,27 & 1,81 & 64 \\
\hline $\mathrm{CUB}_{\mathrm{N}}$ & $C U B_{N}=152,9293^{* *}+182,4446^{* *} D-41,4509^{* *} D^{2}$ & 0,68 & 28,56 & 148 \\
\hline $\mathrm{CUB}_{\mathrm{P}}$ & $C U B_{P}=1377,7710^{* *}+1260,0660^{* *} D$ & 0,80 & 554,96 & 236 \\
\hline $\mathrm{CUB}_{\mathrm{K}}$ & $C U B_{K}=285,4072^{* *}+51,2494^{* *} D$ & 0,54 & 40,64 & 158 \\
\hline $\mathrm{CUB}_{\mathrm{Ca}}$ & $C U B_{C a}=87,9268^{* *}+41,1686^{* *} D-14,3676^{* *} D^{2}$ & 0,69 & 8,66 & 112 \\
\hline $\mathrm{CUB}_{\mathrm{Mg}}$ & $C U B_{M \sigma}=740,3215^{* *}+102,9342^{* *} D$ & 0,46 & 94,72 & 183 \\
\hline $\mathrm{CUB}_{\mathrm{S}}$ & $C U B_{S}=2136,4610^{* *}+716,9103^{*} D-216,2608^{*} D^{2}$ & 0,30 & 207,03 & 207 \\
\hline \multicolumn{5}{|c|}{ 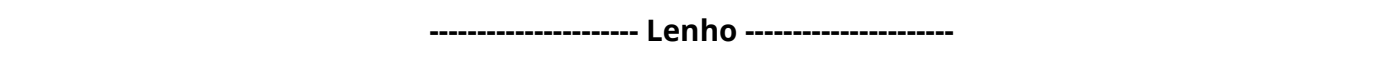 } \\
\hline Bio & Biomassa $=171,4164^{* *}-12,4395^{* *} D$ & 0,46 & 11,55 & 120 \\
\hline $\mathrm{CUB}_{\mathrm{N}}$ & $C U B_{N}=1772,6217^{* *}-204,9828^{* *} D$ & 0,95 & 41,27 & 158 \\
\hline $\mathrm{CUB}_{\mathrm{P}}$ & $C U B_{P}=8738,1850^{* *}+3343,5700^{* *} D-866,6990^{* *} D^{2}$ & 0,89 & 229,03 & 210 \\
\hline $\mathrm{CUB}_{\mathrm{K}}$ & $C U B_{K}=1167,4127^{* *}+172,0823^{* *} D-62,0527^{* *} D^{2}$ & 0,84 & 25,89 & 145 \\
\hline $\mathrm{CUB}_{\mathrm{Ca}}$ & $C U B_{C a}=860,8251^{* *}+1235,4041^{* *} D-301,3237^{* *} D^{2}$ & 0,95 & 58,89 & 169 \\
\hline $\mathrm{CUB}_{\mathrm{Mg}}$ & $C U B_{M \sigma}=8714,9219^{* *}+2172,4792^{* *} D-639,4465^{* *} D^{2}$ & 0,83 & 203,74 & 207 \\
\hline $\mathrm{CUB}_{\mathrm{S}}$ & $C U B_{S}=3743,5044^{* *}-297,1221^{* *} D$ & 0,80 & 132,97 & 193 \\
\hline \multicolumn{5}{|c|}{ - } \\
\hline Bio & Biomassa $=186,3828^{* *}-13,7741^{* *} D$ & 0,44 & 13,22 & 124 \\
\hline $\mathrm{CUB}_{\mathrm{N}}$ & $C U B_{N}=1010,3905^{* *}+187,7565^{* *} D-68,7913^{* *} D^{2}$ & 0,88 & 25,60 & 145 \\
\hline $\mathrm{CUB}_{\mathrm{P}}$ & $C U B_{P}=6077,7875^{* *}+3361,4689^{* *} D-690,6219^{* *} D^{2}$ & 0,93 & 259,91 & 214 \\
\hline $\mathrm{CUB}_{\mathrm{K}}$ & $C U B_{K}=893,3038^{* *}+206,7482^{* *} D-57,6753^{* *} D^{2}$ & 0,80 & 19,73 & 137 \\
\hline $\mathrm{CUB}_{\mathrm{Ca}}$ & $C U B_{C a}=535,6933^{* *}+411,1093^{* *} D-117,7325^{* *} D^{2}$ & 0,86 & 33,22 & 152 \\
\hline $\mathrm{CUB}_{\mathrm{Mg}}$ & $C U B_{M s}=4135,2596^{* *}+1707,3107^{* *} D-388,1108^{* *} D^{2}$ & 0,90 & 134,06 & 194 \\
\hline $\mathrm{CUB}_{\mathrm{S}}$ & $C U B_{S}=3260,3129^{* *}+274,8172^{* *} D-152,8636^{* *} D^{2}$ & 0,91 & 79,45 & 178 \\
\hline
\end{tabular}

*, ** significativo a 5 e $1 \%$ de probabilidade pelo teste $\mathrm{t}$, respectivamente. Bio = biomassa. $\bar{R}^{2}=$ coeficiente de determinação ajustado. Syx = erro-padrão da estimativa. AIC = valor do critério de informação de informação de Akaike. 
A quantidade de biomassa por hectare aumentou com a redução da distância entre plantas. Apesar da significância estatística do coeficiente angular das equações para a estimativa de biomassa (Tabela 3), notou-se pouca diferença em termos produtivos entre os tratamentos de T1 a T4 aos 101 meses de idade (Figura 2). Estes resultados demonstram que se não houver a colheita das árvores no momento da estagnação da taxa de crescimento, com o passar do tempo todos os espaçamentos expressarão a mesma produção final, condizente com Resquin et al. (2019). Quando se opta por diferentes espaçamentos o silvicultor deve se atentar para a adequada idade de corte, rotações mais curtas são indicadas em espaçamentos mais adensados.
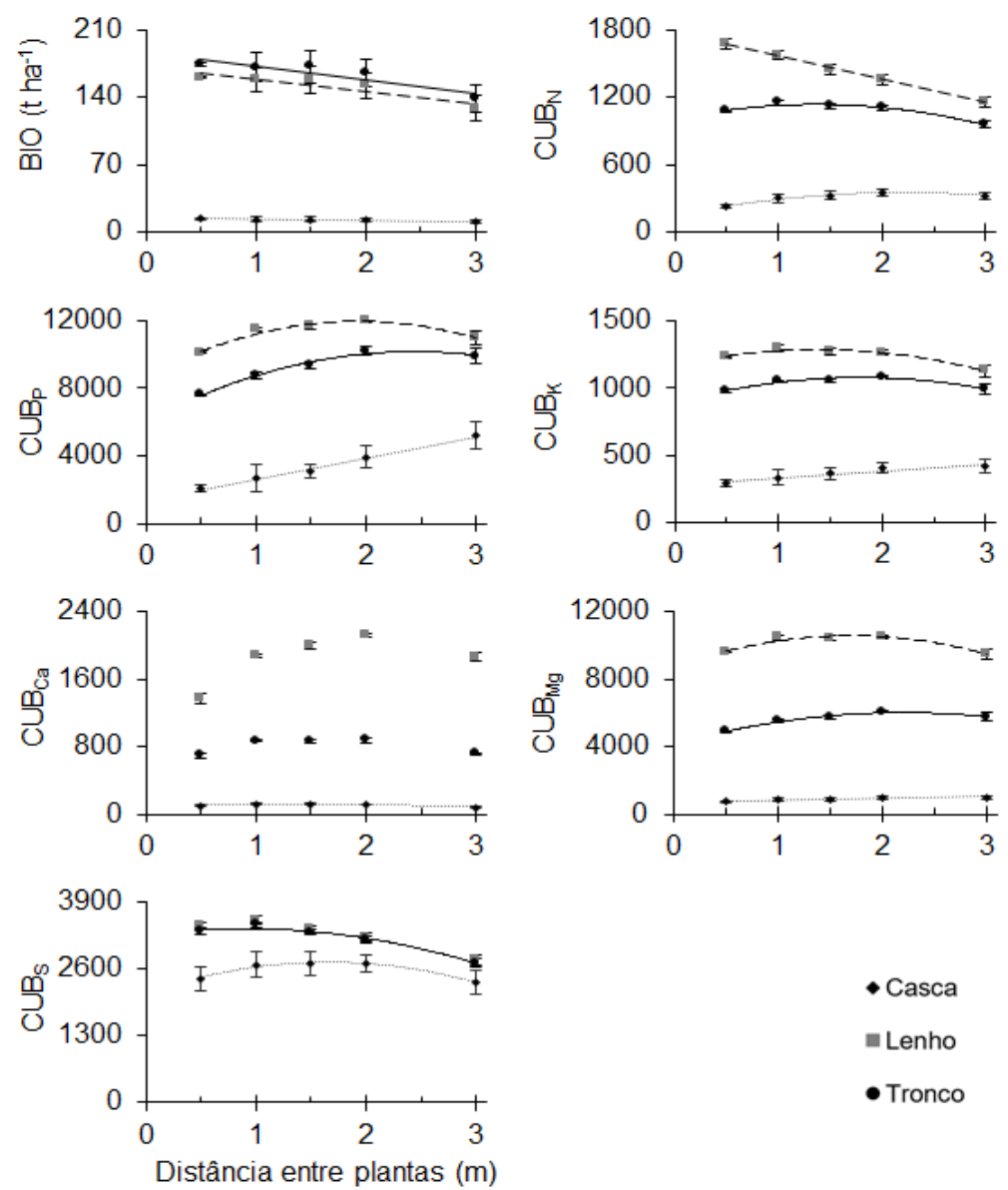

$$
\begin{aligned}
& \text { - Casca } \\
& \text { - Lenho } \\
& \text { - Tronco }
\end{aligned}
$$

Figura 2. Biomassa (BIO) e Coeficiente de Utilização Biológico (CUB) de nutrientes em função da distância entre plantas. A distância entre linhas de plantio foi de $3 \mathrm{~m}$ e as barras de erro referem-se a uma unidade de desvio-padrão.

Os espaçamentos mais adensados foram os mais produtivos; o acréscimo em produção de madeira observada entre os espaçamentos mais adensado $(3,0 \times 0,5 \mathrm{~m})$ e o convencional $3,0 \times 2,0 \mathrm{~m}$ foi de 5,50\% (Figura 2). Entretanto, a viabilidade econômica e ambiental de se adotar curtas rotações deve ser avaliada com cautela e por região, considerando todos os custos e não somente em função da produção. Em muitas ocasiões o aumento de produção não é suficiente para melhorar a viabilidade econômica do projeto, pois os custos de implantação e colheita podem ter impactos negativos em virtude da grande quantidade plantas e fustes de pequenas dimensões (Martins et al., 2009).

O comportamento quadrático foi observado em todas as equações geradas para a estimativa do CUB de nutrientes no tronco em função da distância entre plantas, com coeficientes de determinação ajustado iguais ou superiores a 0,8. Os valores críticos de CUB definidos pelo NUTRICALC, resultado de diversas pesquisas conduzidas em empresas associadas à Sociedade de Investigações Florestais (Barros et al., 1995; Novais et al., 2007) 
para produção de biomassa do tronco de eucalipto são: $\mathrm{P}=12000, \mathrm{~K}=1000, \mathrm{Ca}=600 \mathrm{e} \mathrm{Mg}=$ $3000 \mathrm{~kg}$ de biomassa de tronco por $\mathrm{kg}$ de nutriente. A amplitude de variação entre unidades experimentais do $C U B_{p}$ foi de 7573 a 10450 e do $C^{C U B_{K}}$ de 948 a 1089 para os diferentes espaçamentos. Logo, é plausível assumir que estes nutrientes não limitaram a produção do povoamento. O CUB $\mathrm{Ca}_{\mathrm{C}}$ variou de 657 a 894 e o $\mathrm{CUB}_{\mathrm{Mg}}$ variou de 4807 a 6118, o que indica que estes foram os elementos que provavelmente limitaram o crescimento na rotação atual e poderão limitar a futura, a não ser que sejam supridos via fertilizante em quantidades que atendam à demanda nutricional da cultura.

Apesar de o experimento ter sido conduzido até idade superior àquela convencionalmente sugerida para a colheita de eucaliptais, exibiu resultados relevantes para o planejamento da fertilização e definição de práticas que reduzem a exportação de nutrientes. Nessa circunstância, a colheita restrita ao lenho torna-se uma alternativa viável para a redução de exportação nutricional e da reposição de Ca e Mg via calagem ou resíduos de siderurgia. Valores semelhantes de CUB encontrados neste estudo foram observados para eucalipto aos 78 meses de idade por Santana et al. (2002) e, em povoamentos não desbastados com 10 anos, por Viera et al. (2015).

Não se tem estabelecida na literatura a eficiência crítica, em kg de matéria seca por kg de nutriente, para o S e para o N pelo eucalipto. Observou-se que o $\mathrm{CUB}_{\mathrm{S}}$ variou de 2611 a 3497 e $\mathrm{CUB}_{\mathrm{N}}$ de 923 a 1174. Ainda, são poucos estudos para o S e esta carência é, em parte, atribuída a sua condição de nutriente acompanhante em fertilizações (sulfato de amônio, superfosfato simples e sulfato de potássio). O valor crítico de eficiência permitiria estimar a quantidade de $\mathrm{S}$ a ser recomendada para se alcançar determinada produtividade em diferentes condições edáficas e climáticas. Quanto ao $\mathrm{N}$, as respostas a aplicação deste nutriente são inconsistentes e, quando se expressa, esta ocorre nos anos iniciais de formação do povoamento (Pulito et al., 2015).

Práticas silviculturais baseadas na densidade de plantio podem influenciar o CUB de nutrientes. Menores variabilidades relativas de CUB na casca, lenho e tronco foram verificadas para o S, Mg e K, respectivamente. Observou-se pouca variação de CUB no tronco entre os espaçamentos $3,0 \times 2,0 \mathrm{~m}$ e 3,0 $\times 3,0 \mathrm{~m}$, mais adotados pelo setor florestal brasileiro. Provavelmente, a plasticidade fenotípica intrínseca ao eucalipto sujeito a um ambiente de intensa competição promoveu o uso diferenciado de nutrientes (Stape et al., 2004, 2010). De acordo com Novais et al. (2007) e Cavalcante Junior et al. (2019), alteraç̃̃es fisiológicas de conversão dos nutrientes em biomassa afetam o CUB. Essa adequação ao ambiente pode ocorrer devido aos processos tanto fisiológicos como morfológicos e moleculares na planta, que são induzidos por uma dada restrição imposta pela competição.

A maioria dos usos da madeira considera apenas o lenho como produto principal, entretanto, a principal prática de colheita adotada no Brasil baseia-se na remoção do tronco. No presente estudo, em média, a casca correspondeu a $7,77 \%$ da matéria seca do tronco ou $12,83 \mathrm{t} \mathrm{ha}^{-1}$. A casca tem sido frequentemente transportada para as indústrias, não sendo utilizada como principal produto. A geração de resíduos provenientes da casca pode se tornar passivos ambientais para empresas do setor.

Se a colheita florestal optasse apenas pelo lenho, a eficiência de uso de todos os nutrientes aumentaria expressivamente (Figura 2). A Tabela 4 apresenta a estimativa da quantidade de nutrientes exportados em diferentes intensidades de colheita florestal. No que se refere a remoção de tronco, a economia para a colheita restrita ao lenho seria de, aproximadamente, 59, 49, 29, 24, 23 e 10\%, respectivamente, para $\mathrm{Ca}, \mathrm{Mg}, \mathrm{N}, \mathrm{P}, \mathrm{K}$, e S, respectivamente.

Igualmente relatado por Habitzreiter et al. (2019), manter o máximo de biomassa possível no sítio permite maior sustentabilidade da produtividade. Manter a casca no sítio pode retornar mais de $45 \%$ do Ca e Mg absorvidos. Por outro lado, espera-se ampla depleção de nutrientes do solo nas ocasiões em que a colheita é direcionada para todo o tronco. A colheita da biomassa de lenho com casca apresentou a seguinte magnitude de remoção de nutrientes: $\mathrm{Ca}>\mathrm{K}>\mathrm{N}>\mathrm{S}>\mathrm{Mg}>\mathrm{P}$. Efetuando-se a colheita apenas do lenho, a magnitude de remoção seria: $\mathrm{K}>\mathrm{N}>\mathrm{Ca}>\mathrm{S}>\mathrm{Mg}>\mathrm{P}$. Estes resultados são relevantes para o planejamento 
da fertilização de próximas rotações, pois retratam quais nutrientes deveriam ser repostos em maior ou menor quantidade conforme a intensidade da colheita de árvores de eucalipto.

Tabela 4. Estimativa da quantidade de nutrientes exportada conforme intensidade da colheita de árvores de eucalipto em diferentes espaçamentos.

\begin{tabular}{ccccccc}
\hline \multirow{2}{*}{ Espaçamentos } & $\mathbf{N}$ & $\mathbf{P}$ & $\mathbf{K}$ & $\mathbf{C a}$ & $\mathbf{M g}$ & $\mathbf{S}$ \\
\cline { 2 - 6 } & \multicolumn{7}{c}{ Colheita restrita ao lenho } \\
\cline { 2 - 6 } $3,0 \times 0,5 \mathrm{~m}$ & 96,02 & 15,90 & 130,68 & 117,38 & 16,80 & 46,70 \\
$3,0 \times 1,0 \mathrm{~m}$ & 100,31 & 13,79 & 121,61 & 84,33 & 15,12 & 44,57 \\
$3,0 \times 1,5 \mathrm{~m}$ & 109,28 & 13,58 & 124,41 & 79,10 & 15,20 & 46,89 \\
$3,0 \times 2,0 \mathrm{~m}$ & 112,39 & 12,73 & 120,67 & 72,05 & 14,51 & 47,42 \\
$3,0 \times 3,0 \mathrm{~m}$ & 110,41 & 11,71 & 114,11 & 69,04 & 13,55 & 46,59 \\
& & Colheita do lenho e casca & & \\
$3,0 \times 0,5 \mathrm{~m}$ & 155,30 & 22,55 & 176,85 & 251,87 & 35,35 & 52,37 \\
$3,0 \times 1,0 \mathrm{~m}$ & 145,21 & 18,97 & 161,51 & 196,16 & 30,84 & 49,60 \\
$3,0 \times 1,5 \mathrm{~m}$ & 150,78 & 17,99 & 161,39 & 196,70 & 29,99 & 51,90 \\
$3,0 \times 2,0 \mathrm{~m}$ & 148,56 & 16,01 & 151,90 & 188,11 & 27,17 & 52,17 \\
$3,0 \times 3,0 \mathrm{~m}$ & 142,04 & 13,70 & 138,56 & 193,76 & 23,92 & 51,00 \\
\hline
\end{tabular}

Valores calculados a partir dos dados observados.

Os resultados obtidos fornecem subsídios para o desenvolvimento de futuras pesquisas sobre o balanço nutricional de plantas e melhor planejamento silvicultural do plantio de eucalipto. Enfatiza-se a importância da regulação florestal no fornecimento contínuo de matéria-prima para uso em processos produtivos. Em muitas ocasiões de rotina, opta-se pelo adiamento ou antecipação do corte de certos talhões para a maximização de retorno financeiro, minimização de custos e atendimento a determinadas restrições que influenciam em decisões do manejador. Baseando na teoria do balanço nutricional (Barros et al., 1995; Novais et al., 2007), a colheita restrita ao lenho reduz a reposição de nutrientes via fertilização, promovendo a sustentabilidade, conservação de nutrientes e água no solo e produtividade do sítio (Eufrade Junior et al., 2016). Assim, a produtividade futura em sítios com limitações de nutrientes mais exportados pode ser comprometida caso não seja adotado um correto manejo florestal e de fertilidade do solo.

\section{CONCLUSÕES}

A influência do espaçamento de plantio no coeficiente de utilização biológico na casca, lenho e tronco de povoamentos de eucalipto, aos 101 meses de idade se diferencia entre nutrientes. O espaçamento mais adensado, de 3,0 $\times 0,5 \mathrm{~m}$, exibiu menores estimativas de CUB para $\mathrm{P}$ e $\mathrm{Mg}$ no lenho. O espaçamento mais amplo, de 3,0 × 3,0 m, apresentou o menor CUB de $\mathrm{S}$ no lenho e o maior CUB de $\mathrm{K}$ na casca. A menor variabilidade de CUB no tronco foi para o K e a maior para o Ca.

O Ca é o nutriente mais exportado com a colheita do tronco de árvores de eucalipto aos 101 meses de idade. $\mathrm{O} \mathrm{K}$ foi o nutriente mais exportado com a colheita restrita ao lenho das árvores.

Espaçamentos adensados apresentam fustes menores e, quando devidamente colhidos na idade técnica de corte, são mais produtivos em unidade de área.

\section{REFERÊNCIAS BIBLIOGRÁFICAS}

Associação Brasileira de Normas Técnicas - ABNT. (2003). NBR 11941: madeira: determinação da densidade básica (6 p.). Rio de Janeiro: ABNT. 
Barros, N. F., Ferreira de Novais, R., Teixeira, J. L., \& Fernandes Filho, E. I. (1995). NUTRICALC 2.0: sistema para cálculo del balance nutricional y recomendación de fertilizantes para el cultivo de eucalipto. Bosque (Valdivia), 16(1), 129-131. http://dx.doi.org/10.4206/bosque.1995.v16n1-16.

Barros, N. F., Novais, R. F., Carmo, D. N., \& Neves, J. C. L. (1986). Classificação nutricional de sítios florestais: descrição de uma metodologia. Revista Árvore, 10(2), 112-120.

Binkley, D., Campoe, O. C., Gspaltl, M., \& Forrester, D. I. (2013). Light absorption and use efficiency in forests: why patterns differ for trees and stands. Forest Ecology and Management, 288, 5-13. http://dx.doi.org/10.1016/j.foreco.2011.11.002.

Binkley, D., Stape, J. L., Bauerle, W. L., \& Ryan, M. G. (2010). Explaining growth of individual trees: light interception and efficiency of light use by Eucalyptus at four sites in Brazil. Forest Ecology and Management, 259(9), 1704-1713. http://dx.doi.org/10.1016/j.foreco.2009.05.037.

Binoti, D. H. B., Binoti, M. L. M. S., Leite, H. G., Gleriani, J. M., \& Ribeiro, C. A. A. S. (2014). Inclusão e influência de características espaciais em modelos de regulação florestal. Cerne, 20(1), 157-164. http://dx.doi.org/10.1590/S0104-77602014000100019.

Binoti, D. H. B., Leite, H. G., Nogueira, G. S., Silva, M. L. M., Garcia, S. L. R., \& Cruz, J. P. (2010). Uso da função Weibull de três parâmetros em um modelo de distribuição diamétrica para plantios de eucalipto submetidos a desbaste. Revista Árvore, 34(1), 147-156. http://dx.doi.org/10.1590/S010067622010000100016.

Campoe, O. C., Stape, J. L., Nouvellon, Y., Laclau, J.-P., Bauerle, W. L., Binkley, D., \& Le Maire, G. (2013b). Stem production, light absorption and light use efficiency between dominant and non-dominant trees of Eucalyptus grandis across productivity gradient in Brazil. Forest Ecology and Management, 288, 14-20. http://dx.doi.org/10.1016/j.foreco.2012.07.035.

Campoe, O. C., Stape, J., Albaugh, T. J., Lee Allen, H., Fox, T. R., Rubilar, R., \& Binkley, D. (2013a). Fertilization and irrigation effects on the tree level aboveground net primary production, light interception and light use efficiency in a loblolly pine plantation. Forest Ecology and Management, 288, 43-48. http://dx.doi.org/10.1016/j.foreco.2012.05.026.

Cavalcante Junior, L. F., Soares, I., Taniguchi, C. A. K., Serrano, L. A. L., \& Melo, D. S. (2019). Nutrient accumulation and nutritional efficiencies of cashew genotypes. Journal of Plant Nutrition, 42(4), 344 350. http://dx.doi.org/10.1080/01904167.2018.1555847.

Empresa Brasileira de Pesquisa Agropecuária - EMBRAPA. (2000). Métodos de análise de tecidos vegetais utilizados na Embrapa Solos (Circular Técnica, No. 6). Rio de Janeiro: Embrapa Solos.

Empresa Brasileira de Pesquisa Agropecuária - EMBRAPA. (2018). Sistema brasileiro de classificação de solos (5. ed.). Brasília: Embrapa.

Eufrade Junior, H. J., Melo, R. X., Sartori, M. M. P., Guerra, S. P. S., \& Ballarin, A. W. (2016). Sustainable use of eucalypt biomass grown on short rotation coppice for bioenergy. Biomass and Energy, 90, 15-21. http://dx.doi.org/10.1016/j.biombioe.2016.03.037.

Ferreira, E. B., Cavalcanti, P. P., \& Nogueira, D. A. (2018). ExpDes.pt: Pacote Experimental Designs (Portuguese). R package version 1.2.0. Vienna: R Foundation for Statistical Computing.

Ferreira, G. W. D., Soares, E. M. B., Oliveira, F. C. C., Silva, I. R., Dungait, J. A. J., Souza, I. F., \& Vergütz, L. (2016). Nutrient release from decomposing Eucalyptus harvest residues following simulated management practices in multiple sites in Brazil. Forest Ecology and Management, 370, 1-11. http://dx.doi.org/10.1016/j.foreco.2016.03.047.

Habitzreiter, T. L., Adami, P. F., Sartor, L. R., Brun, E. J., Batista, V. V., \& Prestes Junior, J. V. (2019). Aboveground biomass production and nutrient accumulation in a five year old Eucalyptus grandis and E. urophylla plantation. Colloquium Agrariae, 15(2), 1-8. http://dx.doi.org/10.5747/ca.2019.v15.n2.a279.

Instituto Nacional de Meteorologia - INMET. (2019). Banco de dados meteorológicos para ensino e pesquisa. Brasília. Recuperado em 30 de agosto de 2019, de http://www.inmet.gov.br/

Köppen, W. (1936). Das geographische system der klimate. Berlin: Gerbrüder Bornträger.

Lafetá, B. O., Santana, R. C., Nogueira, G. S., Neves, J. C. L., \& Penido, T. M. A. (2018). Eficiência de utilização de macronutrientes em eucalipto por método não destrutivo estimados por redes neurais artificiais. Ciência Florestal, 28(2), 613-623. http://dx.doi.org/10.5902/1980509832049.

Leslie, A. D., Mencuccini, M., Perks, M. P., \& Wilson, E. R. (2020). A review of the suitability of eucalypts for short rotation forestry for energy in the UK. New Forests, 51(1), 1-19.

http://dx.doi.org/10.1007/s11056-019-09717-w. 
Martins, G. S., Freitas, N. C., Máximo, W. P. F., \& Paiva, L. V. (2018). Gene expression in two contrasting hybrid clones of Eucalyptus camaldulensis $x$ Eucalyptus urophylla grown under water deficit conditions. Journal of Plant Physiology, 229, 122-131. PMid:30071503. http://dx.doi.org/10.1016/j.jplph.2018.07.007.

Martins, R. J., Seixas, F., \& Stape, J. L. (2009). Avaliação técnica e econômica de um harvester trabalhando em diferentes condições de espaçamento e arranjo de plantio em povoamento de eucalipto. Scientia Forestalis, 37(83), 253-263.

Müller, M. D., Couto, L., Leite, H. G., \& Brito, J. O. (2005a). Avaliação de um clone de eucalipto estabelecido em diferentes densidades de plantio para produção de biomassa e energia. Biomassa \& Energia, 2(3), 177-186.

Müller, M. D., Couto, L., \& Neves, J. C. L. (2005b). Produção de biomassa e balanço nutricional de plantações de eucalipto clonal em diferentes densidades de plantio no município de Itamarandiba MG. Biomassa \& Energia, 2(2), 91-101.

Novais, R. F., Alvarez, V. H., Barros, N. F., Fontes, R. L. F., Cantarutti, R. B., \& Neves, J. C. L. (2007). Fertilidade do solo (1. ed.). Viçosa: Sociedade Brasileira de Ciência do Solo.

Pulito, A. P., Gonçalves, J. L. M., Smethurst, P. J., Arthur Junior, J. C., Alvares, C. A., Rocha, J. H. T., Hübner, A., Moraes, L. F., Miranda, A. C., Kamogawa, M. Y., Gava, J. L., Chaves, R., \& Silva, C. R. (2015). Available nitrogen and responses to nitrogen fertilizer in Brazilian eucalypt plantations on soils of contrasting texture. Forests, 6(12), 973-991. http://dx.doi.org/10.3390/f6040973.

R Core Team. (2018). R: a language and environment for statistical computing. Vienna: R Foundation for Statistical Computing.

Resquin, F., Navarro-Cerrillo, R. M., Carrasco-Letelier, L., \& Casnati, C. R. (2019). Influence of contrasting stocking densities on the dynamics of above-ground biomass and Wood density of Eucalyptus benthamii, Eucalyptus dunnii, and Eucalyptus grandis for bioenergy in Uruguay. Forest Ecology and Management, 438, 63-74. http://dx.doi.org/10.1016/j.foreco.2019.02.007.

Rocha, M. F. V., Vital, B. R., Carneiro, A. C. O., Carvalho, A. M. M. L., Cardoso, M. T., \& Hein, P. R. G. (2016). Effect of plant spacing on the physical, chemical and energy properties of Eucalyptus wood and bark. Journal of Tropical Forest Science, 28(3), 243-248.

Santana, R. C., Barros, N. F., \& Neves, J. C. L. (2002). Eficiência de utilização de nutrientes e sustentabilidade da produção em procedências de Eucalyptus grandis e Eucalyptus saligna em sítios florestais do estado de São Paulo. Revista Árvore, 26(4), 447-457. http://dx.doi.org/10.1590/S010067622002000400007.

Schumacher, F. X., \& Hall, F. S. (1933). Logarithmic expression of timber-tree volume. Journal of Agricultural Research, 47(9), 719-734.

Schwerz, F., Eloy, E., Elli, F. E., \& Caron, B. O. (2019). Reduced planting spacing increase radiation use efficiency and biomass for energy in black wattle plantations: towards sustainable production systems. Biomass and Bioenergy, 120, 229-239. http://dx.doi.org/10.1016/j.biombioe.2018.11.034.

Spinelli, R., Ward, S. M., \& Owende, P. M. (2009). A harvest and transport cost model for Eucalyptus spp. fast-growing short rotation plantations. Biomass and Bioenergy, 33(9), 1265-1270. http://dx.doi.org/10.1016/j.biombioe.2009.05.010.

Stape, J. L., Binkley, D., \& Ryan, M. G. (2004). Eucalyptus production and the supply, use and efficiency of use of water, light and nitrogen across a geographic in Brazil. Forest Ecology and Management, 193(12), 17-31. http://dx.doi.org/10.1016/j.foreco.2004.01.020.

Stape, J. L., Binkley, D., Ryan, M. G., Fonseca, S., Loos, R. A., Takahashi, E. N., Silva, C. R., Silva, S. R., Hakamada, R. E., Ferreira, J. M. A., Lima, A. M. N., Gava, J. L., Leite, F. P., Andrade, H. B., Alves, J. M., Silva, G. G. C., \& Azevedo, M. R. (2010). The Brazil Eucalyptus potential productivity project: influence of water, nutrients and stand uniformity on Wood production. Forest Ecology and Management, 259(9), 1684-1694. http://dx.doi.org/10.1016/j.foreco.2010.01.012.

Tavares, A., Beiroz, W., Fialho, A., Frazão, F., Macedo, R., Louzada, J., \& Audino, L. (2019). Eucalyptus plantations as hybrid ecosystems: implications for species conservation in the Brazilian Atlantic forest. Forest Ecology and Management, 433, 131-139. http://dx.doi.org/10.1016/j.foreco.2018.10.063.

Tenorio, C., Moya, R., \& Arias-Aguilar, D. (2018). Evaluation of changes in tree morphology parameters, biomass yield, chemical and energy properties at three spacings of short rotation energy plantations of Gmelina arborea in Costa Rica, from 1 to 2 years of age. Waste and Biomass Valorization, 9(7), 1163-1179. http://dx.doi.org/10.1007/s12649-017-9896-y.

Venables, W. N., \& Ripley, B. D. (2002). Modern applied statistics with S (4th ed.). New York: Springer. http://dx.doi.org/10.1007/978-0-387-21706-2. 
Viera, M., Schumacher, M. V., Trüby, P., \& Araújo, E. F. (2015). Implicações nutricionais com base em diferentes intensidades de colheita da biomassa de Eucalyptus urophylla $\times$ Eucalyptus globulus. Ciência Rural, 45(3), 432-439. http://dx.doi.org/10.1590/0103-8478cr20120367.

Weisberg, S. (2018). Package 'alr3'. R package version 2.0.8. Vienna: R Foundation for Statistical Computing.

Zhou, X., Zhu, H., Wen, Y., Goodale, U. M., Li, X., You, Y., Ye, D., \& Liang, H. (2018). Effects of understory management on trade-offs and synergies between biomass carbon stock plant diversity and timber production in Eucalyptus plantations. Forest Ecology and Management, 410(15), 164-173. http://dx.doi.org/10.1016/j.foreco.2017.11.015.

Contribuição dos Autores: BOL: Conceituação, Curadoria de Dados, Análise Formal, Investigação, Metodologia, Visualização, Escrita - Primeira Redação. RCS: Conceituação, Supervisão, Investigação, Administração do Projeto, Escrita - Revisão e Edição. GSN: Conceituação, Investigação, Escrita - Revisão e Edição. TMAP: Investigação, Análise Formal, Metodologia, Escrita - Revisão e Edição. LFRO: Recursos, Escrita - Revisão e Edição. DSV: Recursos, Escrita - Revisão e Edição. 